\title{
Current clinical use of intravenous fosfomycin in ICU patients in two European countries
}

\author{
C. Putensen ${ }^{1} \cdot$ B. Ellger ${ }^{2}$ S. G. Sakka ${ }^{3} \cdot$ A. Weyland ${ }^{4} \cdot$ K. Schmidt $^{5} \cdot$ M. Zoller ${ }^{6} \cdot$ N. Weiler ${ }^{7} \cdot$ D. Kindgen-Milles ${ }^{8}$.

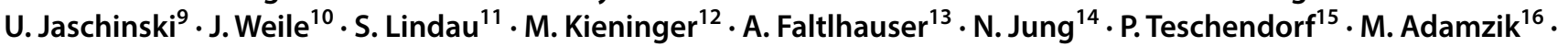 \\ M. Gründling ${ }^{17} \cdot$ T. Wahlers $^{18} \cdot$ H. Gerlach ${ }^{19} \cdot$ F.-A. Litty ${ }^{20}$
}

Received: 3 December 2018 / Accepted: 16 May 2019 / Published online: 12 June 2019

(c) The Author(s) 2019

\begin{abstract}
Purpose In Europe, intravenous fosfomycin (IV) is used particularly in difficult-to-treat or complex infections, caused by both Gram-positive and Gram-negative pathogens including multidrug-resistant strains. Here, we investigated the efficacy and safety of intravenous fosfomycin under real-life conditions.

Methods Prospective, multi-center, and non-interventional study in patients with bacterial infections from 20 intensive care units (ICU) in Germany and Austria (NCT01173575).

Results Overall, 209 patients were included ( 77 females, 132 males, mean age: $59 \pm 16$ years), 194 of which were treated in intensive care (APACHE II score at the beginning of fosfomycin therapy: $23 \pm 8$ ). Main indications ( \pm bacteremia or sepsis) were infections of the CNS (21.5\%), community- (CAP) and hospital-acquired pneumonia (HAP)/ventilator-associated pneumonia (VAP, 15.3\%), bone and joint infections (BJI, 11\%), abdominal infections (11\%), and bacteremia (10.5\%). Most frequently identified pathogens were S. aureus (22.3\%), S. epidermidis (14.2\%), Enterococcus spp. (10.8\%), E. coli (12.3\%) and Klebsiella spp. (7.7\%). At least one multidrug-resistant (MDR) pathogen was isolated from 51 patients (24.4\%). Fosfomycin was administered with an average daily dose of $13.7 \pm 3.5 \mathrm{~g}$ over $12.4 \pm 8.6$ days, almost exclusively (99\%) in combination with other antibiotics. The overall clinical success was favorable in $81.3 \%$ (148/182) of cases, and in $84.8 \%$ (39/46) of patients with $\geq 1$ MDR pathogen. Noteworthy, 16.3\% (34/209) of patients developed at least one, in the majority of cases non-serious, adverse drug reaction during fosfomycin therapy.

Conclusion Our data suggest that IV fosfomycin is an effective and safe combination partner for the treatment of a broad spectrum of severe bacterial infections in critically ill patients.
\end{abstract}

Keywords Intravenous fosfomycin · Non-interventional study $\cdot$ Intensive care $\cdot$ Gram-positive $\cdot$ Gram-negative $\cdot$ Multidrug resistance

\section{Introduction}

Fosfomycin is a bactericidal broad-spectrum antibiotic active against a variety of Gram-positive (GP) and Gramnegative (GN) pathogens including 'problem bacteria' such as carbapenemase- or extended-spectrum beta-lactamase (ESBL)-producing Enterobacteriaceae, methicillin-resistant Staphylococcus aureus (MRSA), glycopeptide-resistant enterococci and multidrug-resistant (MDR) Pseudomonas aeruginosa [1-5], supported by synergistic effects with

F.-A. Litty

felix-alexander.litty@infectopharm.com

Extended author information available on the last page of the article many other antibiotics $[6,8]$. Due to the unique chemical structure, cross-resistance and -allergies with other antibiotics have not been reported so far [7]. Fosfomycin exhibits advantageous pharmacokinetic properties making it particularly useful for complicated deep-seated, even biofilmassociated infections such as respiratory tract, bone or central nervous system (CNS) infections [7, 9-14]. Fosfomycin can be used without any age limitations. However, due to the high sodium load ( $1 \mathrm{~g}$ fosfomycin contains $14 \mathrm{mmol}$ (320 mg) sodium), electrolyte imbalances such as hypernatremia or hypokalemia might occur $[6,15]$.

Fosfomycin was included in the WHO list of essential medicines for treatment of adults and children within the RESERVE group of antibacterial medicines [16, 17]. 
Prospective, randomized, controlled clinical trials with fosfomycin in mono- and combination therapy, though having been initiated recently, are still ongoing. Currently, data on the efficacy and safety of intravenous (IV) fosfomycin in the daily clinical practice are still limited. In addition, fosfomycin is used for indications for which no randomized controlled trials would likely to be feasible due to constraints regarding time and study design.

Here, we present real-life efficacy and safety data on the current clinical use of IV fosfomycin in two countries with longstanding experience from the largest prospective noninterventional study conducted to date.

\section{Patients and methods}

\section{Description of the study}

The present trial is a prospective, open-label, multi-center, and non-interventional study (NIS-FOM; NCT01173575). According to international recommendations, fosfomycin was administered or prescribed based on license approval [18]. The unbiased assignment of a patient to a specific therapeutic strategy was made in best knowledge by investigators in advance, and always met current clinical practice and standards.

Of 25 centers which agreed to participate, 20 recruited patients between June 2010 and June 2016. Database lock was in November 2016 after finalization of documentation of the last patient including queries in October 2016.

The NIS-FOM study including ICF was approved by the ethics committees of participating study centers. Before approval by each study center, the study was approved on 20th August 2010 (Bonn, referral no. 140/10) by the ethics committee at the "coordinating" investigator's study center who signed the study protocol (Medical Department of the University of Bonn). Before inclusion into the study written informed consent was obtained from every single patient or patient's parent(s)/legal guardian(s).

It was the responsibility of the study sites and/or health insurances to bear or reimburse all costs of antimicrobial therapy including IV fosfomycin within the scope of the study, as this reflected antibiotic standard of care within the study center. There was no funding by the manufacturer with regards to study medication. Despite this, every study center received an appropriate and defined expense allowance for each patient documentation including queries.

\section{Patient enrollment and populations}

All consecutive patients of the participating study centers who received treatment with IV fosfomycin were enrolled, provided that informed consent was obtained. Treatment with fosfomycin and the course of infection were followed in terms of clinical and microbiological treatment success and safety until discharge or death, if possible. Any indication for fosfomycin treatment was sufficient for inclusion of patients. Patient enrollment was delayed due to an IV fosfomycin shortage from 2011 to 2013.

All documented patients who received at least one dose of intravenous fosfomycin comprise the intention-to-treat (ITT) population or 'safety population'. All documented patients who received at least one dose of IV fosfomycin and had an evaluable clinical efficacy endpoint form the clinical per protocol (cPP) population or 'clinical effectiveness population'. All documented patients who received at least one dose of IV fosfomycin and had an evaluable microbiological efficacy endpoint form the microbiological per protocol (mPP) population or 'microbiological effectiveness population'.

\section{Recorded data}

An internet-based electronic case report form (eCRF) was used to gather data on the clinical and microbiological efficacy of fosfomycin after intravenous therapy. All data were (pseudo)-anonymized by the investigators at each study center and verified by double-entry procedure.

Recorded data included demographic characteristics, admission and definite diagnosis, height, weight, body mass index (BMI), medical history and treatment indication, intensive care, fosfomycin dose, duration and dosing schedule, pathogens isolated, susceptibility pattern and MDR status, diagnostic methods with respect to infection, relevant concomitant diseases and risk factors, laboratory parameters, APACHE score at baseline and after fosfomycin treatment, body temperature, antimicrobial pretreatment, concomitant antimicrobial agents and duration of hospital stay.

\section{Microbiology and culture procedures}

All microbiological, culture and susceptibility testing procedures were performed according to the local standard operating procedures of the respective centers.

\section{Definitions and outcomes}

Overall clinical success was defined either as clinical cure or clinical improvement. Microbiological success was defined as eradication of the underlying pathogen.

Clinical cure was defined as resolution of signs and symptoms of infection and/or no additional antibiotic therapy necessary. Clinical improvement was defined as improvement of signs and symptoms of infection and/or improvement of signs and symptoms of infection and administration of additional antibiotic therapy. 
Table 1 Description of the demographic and baseline characteristics

\begin{tabular}{lll}
\hline Characteristic & $n$ & $\%$ \\
\hline Age (years) & $59.1 \pm 16.4$ & $63.2 / 36.8$ \\
Gender (m/w) & $132 / 77$ & \\
BMI $\left(\mathrm{kg} / \mathrm{m}^{2}\right)$ & $28.3 \pm 6.9^{*}$ & 92.8 \\
Intensive care patients & 194 & 65.1 \\
Patients with risk factors for bacterial infections & 136 & 73.2 \\
APACHE II (III) score at begin of fosfomycin therapy & $23 \pm 8^{\#} / 104 \pm 18^{* * *}$ & 71.8 \\
Patients with antibiotic pretreatment & 153 & \\
Patients with identified pathogen before start of fosfomycin & 150 & \\
therapy & & \\
\hline Data are mean \pm standard deviation; * 192 patients; ${ }^{*}$ APACHE II (in 71 patients); ** APACHE III (in 19 &
\end{tabular}

Criteria for evaluation included efficacy and safety measurements. The primary efficacy endpoint was clinical success. Secondary efficacy endpoints included the number of patients with cure of infection, improvement of infection, unaltered infection, clinical treatment failure, pathogen eradicated, and microbiological treatment failure.

Clinical efficacy outcome variables were clinical/ microbiological efficacy on day $7 \pm 1$, day $14 \pm 1$ and at discharge. The primary efficacy outcome variable was the efficacy at the first assessment day after end of fosfomycin treatment. For cases with missing data, the last observation was carried forward.

For safety analysis the number of patients with adverse events (AEs), adverse drug reactions (ADRs), serious adverse events (SAEs), number of deaths, and tolerability were recorded.

The term 'difficult-to-treat' infection was used according to common definitions in the literature for deep-seated infections associated with a poor penetration of antibiotics (e.g., CNS infections, bone and joint infections or foreign body associated infections with or without abscess involvement), infections where previous antibiotic (standard) treatment has already failed or infections in patients with high risk of complications (co-morbidities, co-medication, and drug allergies) $[19,20]$.

\section{Statistical analysis}

The statistical analysis was performed using the statistical software SPSS v. 23.0 (IBM). Continuous variables were expressed by mean \pm standard deviation or median and interquartile range (IQR) as appropriate.

\section{Results}

Overall, 209 patients were included (ITT population), for which an evaluable clinical endpoint was attained in 182 cases (cPP population) and an evaluable microbiological endpoint in 90 cases (mPP population). In detail, 172 of the patients were enrolled in 19 German study centers and 37 in one Austrian study center.

\section{Demographic and baseline characteristic}

Patients enrolled were predominantly critically ill, with an APACHE II (III) score at the beginning of fosfomycin treatment of $23 \pm 8$ (data from 71 patients). Virtually all patients were treated in intensive care $(92.8 \%)$, while $65.1 \%(n=136)$ had pre-existing risk factors for bacterial infections such as diabetes mellitus, smoking, immunosuppression/corticosteroids, chronic renal insufficiency, liver cirrhosis and intensive antibiotic pretreatment within the last month. Most patients (71.8\%) had received a targeted therapy and 73.2\% $(n=153)$ a prior therapy with other antibiotics. The patients' demographic and clinical characteristics are presented in Table 1.

\section{Description of the infection and microbiological data}

Main indications for fosfomycin administration were infections of the CNS $(n=45,21.5 \%)$, pneumonia including CAP, HAP and VAP $(n=32,15.3 \%)$, BJI $(n=23,11 \%)$, abdominal infection (SAI, $n=23,11 \%$ ), endocarditis (all with sepsis or bacteremia, $n=9,4.3 \%$ ), complicated urinary tract infection (cUTI, $n=8,3.8 \%)$ and sepsis or bacteremia $(n=22$, $10.5 \%$ ), half of them just with signs of infection or bacteremia but unknown focus. Multiple infections with more than one focus or infections that could not be assigned to one of the other categories were found in $33(15.8 \%)$ patients (see Fig. 1). Overall, 57 (27.3\%) patients developed a clinically and microbiologically confirmed sepsis or bacteremia during 


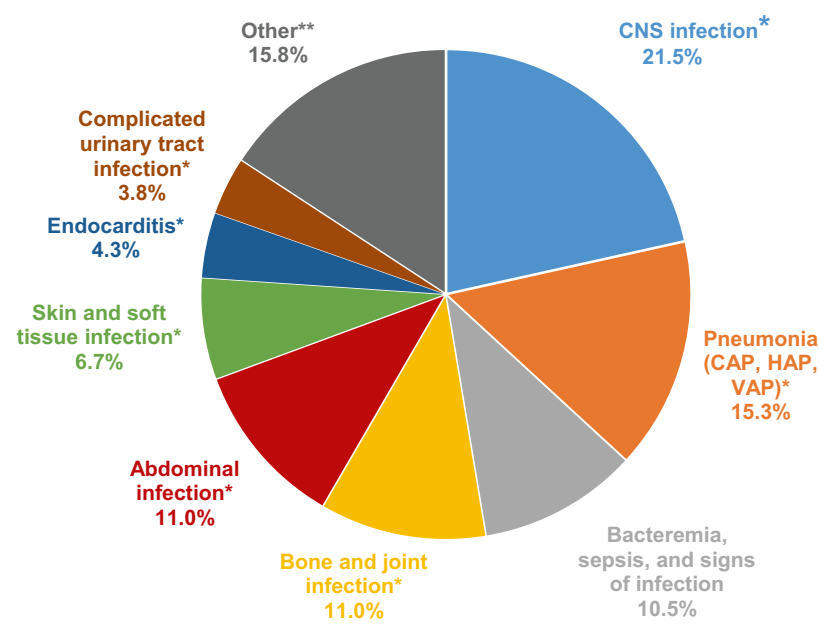

Fig. 1 Types of infection $(n=209)$; \pm bacteremia or sepsis; $* *$ multiple infections or infections that could not be assigned to one of the other categories

Table 2 Microbiological spectrum (data from mono- and polybacterial infections)

\begin{tabular}{lr}
\hline Pathogen & $n(\%)$ \\
\hline Staphylococcus aureus & $58(22.3)$ \\
$\begin{array}{l}\text { Staphylococcus epidermidis/coagulase-negative } \\
\text { staphylococci }\end{array}$ & $37(14.2)$ \\
Escherichia coli & $32(12.3)$ \\
Enterococcus spp. & $28(10.8)$ \\
Klebsiella spp. & $20(7.7)$ \\
Enterobacter/Citrobacter spp. & $17(6.5)$ \\
Pseudomonas aeruginosa & $12(4.6)$ \\
Streptococcus spp. & $9(3.5)$ \\
Proteus spp. & $6(2.3)$ \\
Serratia spp. & $4(1.5)$ \\
Other Gram-negative pathogens & $19(7.3)$ \\
Other Gram-positive pathogens & $9(3.6)$ \\
Anaerobes & $9(3.5)$ \\
\hline
\end{tabular}

the course of infection. One or at least two organ systems ( \pm bacteremia) were affected in $161(77 \%)$ and $37(17.7 \%)$ patients, respectively.

Many infections were polybacterial $(n=77,36.8 \%)$, whereas 60 patients $(28.7 \%)$ had infections by only one pathogen. In 72 (34.4\%) patients, no bacteria or other definite causality for infection could be identified. The majority of pathogens was GP (141 isolates, $54.2 \%$ ). GN pathogens were found in 110 isolates (42.3\%) and other anaerobes in 9 isolates (3.5\%). Fungi were found in 31 patients (14.8\%). The full microbiological spectrum of the 260 isolates is shown in Table 2.
In total, 51 patients $(24.4 \%)$ presented infections due to at least one MDR pathogen. ESBL-producing bacteria were found in four isolates. The resistance pattern of $S$. aureus was not consistently reported for all isolates. In detail, only 28 monobacterial S. aureus infections were assessable, 75\% (21/28) caused by methicillin-sensitive strains (MSSA), while MRSA was isolated in 25\% (7/28) of cases.

\section{Mode and duration of therapy}

The average daily fosfomycin dose was $13.7 \pm 3.5 \mathrm{~g}$, generally split into $2-4$ single doses. Total daily fosfomycin doses ranged between 3 and $24 \mathrm{~g}$, as indicated. The majority of patients $(n=166)$ received $15 \mathrm{~g}$ per day or less (standard dose group), and 41 patients received more than $15 \mathrm{~g}$ per day (high-dose group). The average fosfomycin dose for patients with infections due to GP pathogens was $13.2 \pm 7.0 \mathrm{~g} / \mathrm{d}$ and $13.6 \pm 6.9$ $\mathrm{g} /$ day for infections due to GN pathogens. For two patients, the fosfomycin dose was not reported. The mean treatment duration was $12.4 \pm 8.6$ days, strongly depending on the indication: average treatment duration was 20 days in BJI and sepsis, and 6 days in cUTI.

Fifty-six patients (26.8\%) received fosfomycin as first-line therapy, particularly for infections of a single organ system $(49 / 161,30.4 \%)$. For infections of two or more organ systems, fosfomycin was used less frequently as the first-line treatment $(6 / 32,6.2 \%)$. As a first-line therapy, fosfomycin was employed most frequently in patients with CNS infections $(25 / 45,55.5 \%)$, followed by cUTI $(4 / 8,50 \%)$, endocarditis $(2 / 9,22.2 \%)$, abdominal infections $(5 / 23,21.7 \%)$, bacterial pneumonia $(6 / 32,18 \%)$, BJI $(4 / 23,17.4 \%)$, and sepsis or bacteremia with unknown entry $(1 / 11,9.1 \%)$. In patients with skin and soft tissue infections (SSTI), fosfomycin was used at least as second-line option.

Fosfomycin was nearly exclusively used in combination therapy (207 patients, 99\%) and predominantly combined with a beta-lactam, glycopeptide, metronidazole and/or a quinolone (Table 3). Most patients were treated with one (80 patients), two (72 patients) or three (30 patients) combination partners. More than three concomitant antibiotics were administered in 25 patients.

Combination regimens of the three most frequently reported indications are shown in Table 4.

A carbapenem was the preferred combination partner for fosfomycin, except in patients with CNS infections. Here, fosfomycin was predominantly combined with a third- or fourthgeneration cephalosporin.

\section{Clinical/microbiological efficacy and emergence of resistance}

Overall clinical success was $81.3 \%$ (Table 5) and microbiological success was $70 \%$ (Table 6). No emergence of 
Table 3 Fosfomycin combination partners with number of patients per class $(n=209)$

\begin{tabular}{lcc}
\hline Combination partner & $\begin{array}{l}\text { Number of } \\
\text { patients }\end{array}$ & $\%$ \\
\hline Carbapenem & 102 & 48.8 \\
Glycopeptide & 66 & 31.6 \\
3rd- or 4th-generation cephalosporin & 58 & 27.8 \\
Penicillins + beta-lactamase inhibitor & 30 & 14.4 \\
Metronidazole & 26 & 12.4 \\
Quinolone & 23 & 11.0 \\
Penicillin & 22 & 10.5 \\
1st- or 2nd-generation cephalosporin & 13 & 6.2 \\
Linezolid & 13 & 6.2 \\
Aminoglycoside & 13 & 6.2 \\
Macrolide & 12 & 5.7 \\
Sulfonamide & 11 & 5.3 \\
Daptomycin & 10 & 4.8 \\
Colistin & 7 & 3.3 \\
Rifampicin & 7 & 3.3 \\
Tigecycline & 6 & 2.9 \\
Other & 9 & 4.3 \\
\hline
\end{tabular}

Table 4 Fosfomycin combination partners in subgroups of patients with bacterial pneumonia, abdominal infection and CNS infection (all without sepsis or bacteremia)

\begin{tabular}{lc}
\hline Combination partner & $\begin{array}{l}\text { Number } \\
\text { of patients } \\
(\%)\end{array}$ \\
\hline Patients with bacterial pneumonia (CAP, HAP or VAP) & $25(100)$ \\
Carbapenem & $15(60.0)$ \\
Glycopeptide & $7(28.0)$ \\
3rd- or 4th-generation cephalosporin & $6(24.0)$ \\
Colistin & $5(20.0)$ \\
Quinolone & $4(16.0)$ \\
Patients with abdominal infection & $22(100)$ \\
Carbapenem & $18(81.8)$ \\
3rd- or 4th-generation cephalosporin & $4(18.2)$ \\
Glycopeptide, penicillin + BLI or metronidazole & $3(13.6)$ \\
Patients with CNS infection & $44(100)$ \\
3rd- or 4th-generation cephalosporin & $34(77.3)$ \\
Carbapenem & $11(25.0)$ \\
Metronidazole & $11(25.0)$ \\
Glycopeptide & $10(22.7)$ \\
\hline
\end{tabular}

$H A P$ hospital-acquired pneumonia; $C A P$ community-acquired pneumonia; $V A P$ ventilator-associated pneumonia; $B L I$ beta-lactamase inhibitor; $C N S$ central nervous system
Table 5 Clinical efficacy endpoints $(n=209)$

\begin{tabular}{lrrr}
\hline Efficacy endpoint & $n$ & $\%$ & $\%(\mathrm{cPP})$ \\
\hline Cure & 63 & 30.1 & 34.6 \\
Improvement & 85 & 40.7 & 46.7 \\
Failure & 19 & 9.1 & 10.4 \\
Unchanged & 15 & 7.2 & 8.3 \\
Not reported & 3 & 1.4 & \\
Not assessable* & 24 & 11.5 & \\
\hline
\end{tabular}

cPP clinical per protocol population

*According to physicians' assessment, no definite classification. Last documented observation carried forward independently of previous assessments at earlier time points (e.g., day 7 or 14)

Table 6 Microbiological efficacy endpoints $(n=209)$

\begin{tabular}{lccl}
\hline Efficacy endpoint & $n$ & $\%$ & $\%(\mathrm{mPP})$ \\
\hline Eradication & 63 & 30.1 & 70 \\
Microbiological treatment & 27 & 12.9 & 30 \\
$\quad$ failure & 3 & 1.5 & \\
Not reported & 47 & 22.5 & \\
Not determined & 69 & 33.0 & \\
Not assessable & &
\end{tabular}

mPP microbiological per protocol population

resistance against fosfomycin was observed during fosfomycin treatment.

In patients with infections of a single organ system, clinical success was achieved in $82.1 \%$ (115/140) of patients. With infections of two or more organ systems, clinical success was just slightly lower $(75 \%, 24 / 32)$. The highest clinical success rate of $90 \%(9 / 10)$ was found in patients with signs of infection or bacteremia, but unknown focus. Eradication could be achieved in all patients with BJI, SSTI and endocarditis. The clinical and microbiological success per indication is shown in Table 7.

Clinical success rates in the standard and high-dose groups were virtually identical $(81.1 \%$ vs. $81.3 \%$ (cPP)). In two patients with clinical cure, fosfomycin dosage was unknown.

In cases involving MDR pathogens, clinical and microbiological success rates were $84.8 \%$ (39/46 (cPP)) and $81.5 \%$ $(22 / 27(\mathrm{mPP}))$, respectively. Clinical and microbiological success rates in infections due to MRSA were $100 \%(6 / 6$ (cPP)) and 66.7\% (4/6 (mPP)), in MSSA 76.2\% (16/21 (cPP)) and 100\% (9/9 (mPP)), respectively.

If fosfomycin was combined with either a carbapenem, cephalosporin or a penicillin, pooled clinical success rates were $80.8 \%, 87.5 \%$, and $85.7 \%$, respectively (Table 8 ). 
Table 7 Clinical and microbiological success per indication

\begin{tabular}{|c|c|c|c|c|}
\hline \multirow[t]{2}{*}{ Indication } & \multicolumn{2}{|c|}{ Clinical success (cPP) } & \multicolumn{2}{|c|}{$\begin{array}{l}\text { Microbiological } \\
\text { success (eradica- } \\
\text { tion, mPP) }\end{array}$} \\
\hline & $n$ & $\%$ & $n$ & $\%$ \\
\hline All patients & $148 / 182$ & 81.3 & $63 / 90$ & 70.0 \\
\hline CNS infections* & $33 / 37$ & 89.1 & $9 / 11$ & 81.8 \\
\hline Pneumonia (CAP, HAP, VAP)* & $23 / 27$ & 85.2 & $7 / 13$ & 53.8 \\
\hline Bacteremia, sepsis, and signs of infection & $17 / 20$ & 85.0 & $7 / 10$ & 70.0 \\
\hline Bacteremia with unknown focus and signs of infection & $9 / 10$ & 90 & $4 / 5$ & 80.0 \\
\hline Bone and join infections* & $18 / 21$ & 85.7 & $13 / 13$ & 100.0 \\
\hline Abdominal infections* & $16 / 22$ & 72.7 & $3 / 10$ & 30.0 \\
\hline Skin and soft tissue infections* & $10 / 12$ & 83.3 & $8 / 8$ & 100.0 \\
\hline Endocarditis** & $6 / 9$ & 66.7 & $4 / 4$ & 100.0 \\
\hline Complicated urinary tract infections* & $6 / 7$ & 85.7 & $5 / 6$ & 83.3 \\
\hline Infection due to MDR pathogens & $39 / 46$ & 84.8 & $22 / 27$ & 81.5 \\
\hline Infection due to GP pathogens ${ }^{\#}$ & $49 / 65$ & 75.4 & $23 / 33$ & 69.7 \\
\hline Infection due to GN pathogens ${ }^{\#}$ & $20 / 25$ & 80.0 & $10 / 13$ & 76.9 \\
\hline Infection due to GP and GN pathogens (polybacterial) & $35 / 37$ & 94.6 & $23 / 21$ & 90.5 \\
\hline Infection due to MSSA & $16 / 21$ & 76.2 & $9 / 9$ & 100 \\
\hline Infection due to MRSA & $6 / 6$ & 100 & $4 / 6$ & 66.7 \\
\hline Infection without identified pathogens & $44 / 55$ & 80.0 & & \\
\hline
\end{tabular}

$M D R$ multidrug-resistant, $G P / G N$ Gram-positive/-negative; $M S$-/MRSA methicillin-susceptible/methicillinresistant $S$. aureus

$*_{ \pm}$Sepsis or bacteremia, $* *$ all with sepsis or bacteremia, ${ }^{*}$ microbiologically confirmed (=targeted therapy)
Table 8 Clinical success of fosfomycin in combination therapy (combinations of fosfomycin with one other concomitant antibiotic only)

\begin{tabular}{|c|c|c|c|}
\hline Class & $n(\mathrm{ITT})$ & $\begin{array}{l}\text { Combination partner } \\
(n \text { ITT })\end{array}$ & $\begin{array}{l}\% \text { Clinical } \\
\text { success } \\
(n \mathrm{cPP})\end{array}$ \\
\hline Carbapenems & 30 & $\begin{array}{l}\text { Meropenem (27) } \\
\text { Imipenem (3) }\end{array}$ & $80.8 \%(21 / 26)$ \\
\hline Cephalosporins & 23 & $\begin{array}{l}\text { Cefotaxime (13) } \\
\text { Cefuroxime (5) } \\
\text { Cefazolin (2) } \\
\text { Ceftriaxone (2) } \\
\text { Ceftazidime (1) }\end{array}$ & $87.5 \%(14 / 16)$ \\
\hline Penicillins & 15 & $\begin{array}{l}\text { Piperacillin/tazobactam } \\
\text { (8) } \\
\text { Flucloxacillin (5) } \\
\text { Amoxicillin/clavulanic } \\
\text { acid (1) } \\
\text { Ampicillin/sulbactam (1) }\end{array}$ & $85.7 \%(12 / 14)$ \\
\hline
\end{tabular}

$n$ no. of patients; ITT intention-to-treat population, $c P P$ clinical per protocol population

\section{Safety}

In total, 70 adverse events (AEs) were reported in 63 patients $(30.1 \%)$. In seven patients, two AEs were reported, but no
Table 9 Summary of safety analysis (adverse events, adverse drug reactions* and death)

\begin{tabular}{lrcc}
\hline Classification & $n$ & $n_{\text {(patients) }}$ & $\%$ (ITT) \\
\hline AE $_{\text {(total) }}$ & 70 & 63 & 30.1 \\
Non-serious ADR & 36 & 31 & 14.8 \\
Serious ADR & 36 & 3 & 1.4 \\
Death & 3 & 15 & 7.2 \\
\hline
\end{tabular}

*Considered as being related to fosfomycin

$A E$ adverse event, $A D R$ adverse drug reaction

patient experienced more than two AEs. Thirty-nine AEs were considered being related to the study drug (adverse drug reactions, ADR), three of which were assessed as serious. One of these three patients developed a multiple organ failure, another an acute respiratory insufficiency in pneumonia and a terminal cystic fibrosis, both with fatal outcome and both considered being unlikely related to fosfomycin. The third serious ADR was a severe hypokalemia that did not lead to discontinuation of fosfomycin treatment, however. A summary of the safety analysis is shown in Table 9 .

Most common adverse events were hypernatremia $(n=31$, $14.8 \%)$ and hypokalemia $(n=13,6.2 \%)$. A causal relationship to fosfomycin was suspected in $22(10.5 \%)$ patients with hypernatremia and five $(2.4 \%)$ with hypokalemia. Of 
Table 10 Overview of adverse events

\begin{tabular}{lcl}
\hline AE & $n_{\text {related }}(\%($ ITT $))$ & $n_{\text {not related }}(\%$ (ITT)) \\
\hline Hypernatremia & $22(10.5 \%)$ & $9(4.3 \%)$ \\
Hypokalemia & $5(2.4 \%)$ & $8(3.8 \%)$ \\
Diarrhea & $3(1.4 \%)$ & \\
Nausea & $2(1 \%)$ & \\
Transaminases increased & $1(0.5 \%)$ & \\
Pyrexia & $1(0.5 \%)$ & \\
Allergic reaction & $1(0.5 \%)$ & \\
Hyponatremia & $1(0.5 \%)$ & $1(0.5 \%)$ \\
Hyperkalemia & & \\
Not specified & $1(0.5 \%)$ & \\
\hline
\end{tabular}

$A E$ adverse event; ITT intention-to-treat population

note, one and six patients, respectively, had hypokalemia or hypernatremia prior to fosfomycin treatment. Only four patients developed hypokalemia concomitantly with hypernatremia. In one patient, occurrence of an $\mathrm{AE}$ was formally documented, but not further specified. An overview of all AEs is shown in Table 10.

In eight patients, fosfomycin treatment was discontinued due to an adverse event, with hypernatremia being the cause in six of these cases. In the remaining two cases, therapy was stopped either due to an allergic reaction or pyrexia and nausea.

\section{Discussion}

To date, this study is the largest non-interventional study focusing on the use of intravenous fosfomycin in the daily clinical practice. In total, 209 critically ill patients were enrolled, presenting with severe, life-threatening infections. Severity of illness of our patients was higher as of those reported in a previous study [39].

In our study, we found a broad use of IV fosfomycin against both GP and GN pathogens with a higher proportion of Gram-positives (54.2\%). The widespread spectrum of indications ranging from infections predominantly caused by Gram-negatives such as cUTI to others most often associated with GP pathogens such as CNS infections, BJI or endocarditis, indicate that fosfomycin has been used across different sites of infection. High in vitro activity against several 'problem bacteria', in particular $S$. aureus and $S$. epidermidis, is clinically reflected by the large number of staphylococcal infections (36.5\%) treated in NIS-FOM [21, 22]. Despite several alternative therapeutic options, fosfomycin was mostly used in MSSA infections [23, 24]. This might suggest that severe $S$. aureus infections with deep focus might represent entities not yet adequately covered by standard therapy [25].
In contrast to studies solely reporting on the use of IV fosfomycin as a last-resort option in infections due to MDR GN pathogens such as carbapenemase-producing Enterobacteriaceae or ESBL-producers [26, 33, 38, 39], data from this two-country study suggest that only in $24 \%$ of all patients the indication for fosfomycin use was an infection caused by MDR pathogens. More often it was used as a combination partner against other difficult-to-treat infections with limited therapeutic options or where the first-line treatment had already failed. In difficult-to-treat infections, even in those with biofilm involvement, IV fosfomycin has become a valuable combination partner supported by its advantageous pharmacokinetic properties [26-28, 30]. Its chemical properties allow fosfomycin to penetrate deep into tissues, even into bones and the CNS, which might explain the preferred use in CNS infections (22.3\%) such as brain abscesses or bacterial meningitis, BJI (14.2\%), or sepsis (10.5\%) [11, 13, 29].

The broad spectrum of combination partners might be due to synergistic or additive effects of fosfomycin with many other classes of antibiotics that could be explained by inhibiting the bacterial cell wall synthesis at an earlier stage than other antibiotics (e.g., beta-lactams) [2]. Interestingly, our data indicate that fosfomycin was not used as a carbapenem-sparing treatment option, evermore discussed in the literature [31, 32]. Instead, in infections like nosocomial pneumonia (HAP, VAP) or abdominal infection (Table 4) fosfomycin was most often combined with a carbapenem. This combination is supported by synergistic effects and has recently been recommended, especially if MDR GN pathogens are involved [26, 33-36].

Although IV fosfomycin is generally considered a second-line treatment option, it was used in first-line therapy in more than a quarter of patients. Given its ability to rapidly cross the blood/brain barrier, fosfomycin may be regarded as a useful combination partner for treatment of CNS infections, e.g., when combined with a third- or fourth-generation cephalosporin [37].

The overall clinical success rate in the NIS-FOM study is the highest compared to other more recent studies on IV fosfomycin with similar design. Dinh et al. reported a favorable overall outcome in $76.8 \%$ of evaluable patients; BJI $82.6 \%$, lung infection $67.7 \%$, UTI $100 \%$, endocarditis or neurological infection $83.3 \%$, and bacteremia $57.1 \%$ [38]. According to Pontikis et al., clinical outcome at day 14 was successful in $54.2 \%$ of patients, whilst failure, indeterminate outcome and superinfection were documented in $33.3 \%, 6.3 \%$ and $6.3 \%$, respectively [39]. However, in both studies the proportion of MDR pathogens was significantly higher, ranging between 70 and $100 \%$. In NIS-FOM, clinical success rates ranged from $67 \%$ in patients with endocarditis to $89 \%$ in patients with CNS infection, emphasizing the role of fosfomycin in the treatment of GP pathogens [6]. Of note, all 
patients with endocarditis developed a bacteremia or sepsis concomitantly, hampering a favorable clinical outcome [40]. In the subgroup of infections with MDR pathogen (including MRSA), clinical and microbiological success was adequate (Table 7). The reason for comparatively low clinical and microbiological success in patients with intraabdominal infections is unknown so far and needs further investigation.

Critically ill patients with impaired organ function and intensive co-medication challenge a safe and effective antibiotic treatment [42]. Pharmacokinetic interactions are common, possibly leading to discontinuation of therapy [43]. Many antibiotics, e.g., glycopeptides or colistin, are associated with serious adverse events such as nephrotoxicity, limiting their therapeutic use [44, 45]. However, our data show that IV fosfomycin was used in a large dosing range. Reported adverse events were generally non-serious and expected, without troubling drug/drug interactions. Moreover, safety results from the NIS-FOM are in line with recent reviews corroborating the good safety profile of the substance $[6,15]$. Given the sodium load of IV fosfomycin, discontinuation of the drug due to accumulating high sodium levels in six cases was reasonable to prevent potentially serious electrolyte imbalances. One limitation of our study is the lack of a control group. Thus, it remains unclear how many patients would have developed hypernatremia or hypokalemia independent of fosfomycin treatment. In general, hypokalemia is a common problem in ICU patients and medication is merely one possible explanation [41]. Therefore, clinicians should be aware of disturbances in sodium and potassium homeostasis and initiate adequate measures early to avoid further complications. Altogether, fosfomycin's safety profile appears advantageous, since the generally non-serious and expected nature of adverse events constitutes no limiting factor in therapy.

\section{Conclusion}

These results suggest that IV fosfomycin is an effective and safe combination partner in the treatment of critically ill patients with severe infections due to Gram-negative and Gram-positive pathogens, even in first-line treatment. Fosfomycin was used across different indications and sites of infection, independently of the pathogen's resistance pattern. On the other hand, IV fosfomycin is also suitable for highly specific patients and settings, when standard treatment might be inadequate due to clinical circumstances or has already failed. Thus, considering the critical medical condition of the study population and the severity of underlying infections, the high overall success rate of $81 \%$ together with an advantageous safety profile is very promising and might give rise for further prospective clinical trials.
Acknowledgements We gratefully acknowledge the NIS-FOM study group for excellent cooperation.

\section{Compliance with ethical standards}

Conflict of interest This study was funded by InfectoPharm Arzneimittel und Consilium GmbH (D) and Sandoz GmbH (AT). All participating study centers received contractually agreed study fees by InfectoPharm Arzneimittel und Consilium GmbH during the conduct of the present study. DKM reports grants from InfectoPharm during the conduct of the study and personal fees outside the submitted work. In the last three years, $\mathrm{NJ}$ has received lecture fees from Labor Stein, Novartis, Gilead, InfectoPharm and MSD and travel grants from Gilead, Novartis and Basilea. FAL is an employee of InfectoPharm Arzneimittel und Consilium GmbH. The other authors have nothing to disclose.

Ethical standards The present study has been approved by the appropriate ethics committee and has, therefore, been conducted in accordance with the ethical standards laid down in the 1964 Declaration of Helsinki and its later amendments.

Open Access This article is distributed under the terms of the Creative Commons Attribution 4.0 International License (http://creativeco mmons.org/licenses/by/4.0/), which permits unrestricted use, distribution, and reproduction in any medium, provided you give appropriate credit to the original author(s) and the source, provide a link to the Creative Commons license, and indicate if changes were made.

\section{References}

1. Hendlin D, Stapley EO, Jackson M, Wallick H, Miller AK, Wolf FJ, Miller TW, Chaiet L, Kahan FM, Foltz EL, Woodruff HB, Mata JM, Hernandez S, Mochales S. Phosphonomycin, a new antibiotic produced by strains of streptomyces. Science. 1969;166:122-3.

2. Kahan FM, Kahan JS, Cassidy PJ, Kropp H. The mechanism of action of fosfomycin (phosphonomycin). Ann N Y Acad Sci. 1974;235:364-86.

3. Falagas ME, Kastoris AC, Kapaskelis AM, Karageorgopoulos DE. Fosfomycin for the treatment of multidrug-resistant, including extended-spectrum $\beta$-lactamase producing, Enterobacteriaceae infections: a systematic review. Lancet Infect Dis. 2010;10:43-50.

4. Falagas ME, Roussos N, Gkegkes ID, Rafailidis PI, Karageorgopoulos DE. Fosfomycin for the treatment of infections caused by Gram-positive cocci with advanced antimicrobial drug resistance: a review of microbiological, animal and clinical studies. Expert Opin Invest Drugs. 2009;18:921-44.

5. Falagas ME, Vouloumanou EK, Samonis G, Vardakas KZ. Fosfomycin. Clin Microbiol Rev. 2016;29:321-47.

6. Grabein B, Graninger W, Rodríguez Baño J, Dinh A, Liesenfeld DB. Intravenous fosfomycin-back to the future. Systematic review and meta-analysis of the clinical literature. Clin Microbiol Infect. 2017;23:363-72.

7. Michalopoulos AS, Livaditis IG, Gougoutas V. The revival of fosfomycin. Int J Infect Dis. 2011;15:e732-9.

8. Kastoris AC, Rafailidis PI, Vouloumanou EK, Gkegkes ID, Falagas ME. Synergy of fosfomycin with other antibiotics for Grampositive and Gram-negative bacteria. Eur J Clin Pharmacol. 2010;66:359-68.

9. Matzi V, Lindenmann J, Porubsky C, Kugler SA, Maier A, Dittrich P, Smolle-Jüttner FM, Joukhadar C. Extracellular concentrations 
of fosfomycin in lung tissue of septic patients. J Antimicrob Chemother. 2010;65:995-8.

10. Bonora V, Lozano C, Santos M, Paz M, Baguena J, Gobernado M. Fosfomycin in treatment of respiratory bacterial infections. Chemotherapy. 1977;23:337-41.

11. Pfausler B, Spiss H, Dittrich P, Zeitlinger M, Schmutzhard E, Joukhadar C. Concentrations of fosfomycin in the cerebrospinal fluid of neurointensive care patients with ventriculostomy-associated ventriculitis. J Antimicrob Chemother. 2004;53:848-52.

12. Tritthart H. Fosfomycin in cerebral and spinal abscesses. In: Guggenbichler JP, editor. New aspects of treatment with fosfomycin. Vienna: Springer; 1987. p. 58-66.

13. Schintler MV, Traunmüller F, Metzler J, Kreuzwirt G, Spendel S, Mauric O, Popovic M, Scharnagl E, Joukhadar C. High fosfomycin concentrations in bone and peripheral soft tissue in diabetic patients presenting with bacterial foot infection. J Antimocrob Chemother. 2009;64:574-8.

14. Meißner A, Haag R, Rahmanzadeh R. Adjuvant fosfomycin medication in chronic osteomyelitis. Infection. 1989;17:146-51.

15. Iarikov D, Wassel R, Farley J, Nambiar S. Adverse events associated with fosfomycin use: review of the literature and analysis of the FDA adverse event reporting system database. Infect Dis Ther. 2015;4:433-58.

16. World Health Organisation. WHO Model List of Essential Medicines. 20th ed. Geneva; March 2017 (Amended August 2017).

17. World Health Organisation. WHO Model List of Essential Medicines for Children. 6th ed. Geneva; March 2017 (Amended August 2017).

18. Paul-Ehrlich-Institut. Empfehlungen des Bundesinstituts für Arzneimittel und Medizinprodukte und des Paul-Ehrlich-Instituts zur Planung, Durchführung und Auswertung von Anwendungsbeobachtungen (AWB) vom 7. Juli 2010.

19. de Marie S. Difficult-to-treat infections. Intensive Care Med. 1990;16:S239-42.

20. Schierholz JM, Beuth J, Pulverer G. "Difficult to treat infections" pharmacokinetic and pharmacodynamic factors-a review. Acta Microbiol Immunol Hung. 2000;47:1-8.

21. Vardakas KZ, Legakisc NJ, Triaridesa N, Falagas ME. Susceptibility of contemporary isolates to fosfomycin: a systematic review of the literature. Int J Antimicrob Agents. 2016;47:269-85.

22. Kresken M, Hafner D, Körber-Irrgang B, Working Party Antimicrobial Resistance of the Paul-Ehrlich-Society for Chemotherapy. Resistenzstudie 2013, Abschlussbericht-Teilprojekt H. Epidemiologie und Resistenzsituation bei klinisch wichtigen Infektionserregern aus dem Hospitalbereich gegenüber Antibiotika. Rheinbach, Germany, 27. Juli 2016.

23. Rayner C, Munckhof WJ. Antibiotics currently used in the treatment of infections caused by Staphylococcus aureus. Intern Med J. 2005;35:S3-16.

24. Bodmann K, Grabein B, Kresken M, Derendorf H, Stahlmann R, Ott SR, Olzowy B, Eckmann C, Wagenlehner F, Sunderkötter C, Voßen M, Dohmen PM, Shah PM, Mutters R, Walger P, Michael Wilke M. Paul Ehrlich Society for Chemotherapy (ed.). S2 k-Leitlinie: Kalkulierte parenterale Initialtherapie bakterieller Erkrankungen bei Erwachsenen - Update 2018. Association of the Scientific Medical Societies. Guideline no. 082-006, 01. Rheinbach, Germany, 2017.

25. Chew R, Woods ML. Flucloxacillin does not achieve therapeutic cerebrospinal fluid levels against meticillin-sensitive Staphylococcus aureus in adults: a case report and review of the literature. Int J Antimicrob Agents. 2016;47:229-31.

26. Bassetti M, Giacobbe DR, Giamarellou H, Viscoli C, Daikos GL, Dimopoulos G, De Rosa FG, Giamarellos-Bourboulis EJ, Rossolini GM, Righi E, Karaiskos I, Tumbarello M, Nicolau DP, Viale PL, Poulakou G. Critically Ill Patients Study Group of the European Society of Clinical Microbiology and Infectious Disease
(ESCMID); Hellenic Society of Chemotherapy (HSC) and Società Italiana di Terapia Antinfettiva (SITA). Management of KPC-producing Klebsiella pneumoniae infections. Clin Microbiol Infect. 2018:24:133-44.

27. Mihailescu R, Furustrand Tafin U, Corvec S, Oliva A, Betrisey B, Borens O, Trampuz A. High activity of fosfomycin and rifampin against methicillin-resistant Staphylococcus aureus biofilm in vitro and in an experimental foreign-body infection model. Antimicrob Agents Chemother. 2014;58:2547-53.

28. Corvec S, Furustrand Tafin U, Betrisey B, Borens O, Trampuz A. Activities of fosfomycin, tigecycline, colistin, and gentamicin against extended-spectrum- $\beta$-lactamase-producing Escherichia coli in a foreign-body infection model. Antimicrob Agents Chemother. 2013;57:1421-7.

29. Kühnen E, Pfeifer G, Frenkel C. Penetration of fosfomycin into cerebrospinal fluid across non-inflamed and inflamed meninges. Infection. 1987;15:422-4.

30. Bandeira M, Carvalho PA, Duarte A, Jordao L. Exploring dangerous connections between Klebsiella pneumoniae biofilms and healthcare-associated infections. Pathogens. 2014;3:720-31.

31. Fournier D, Chirouze C, Leroy J, Cholley P, Talon D, Plésiat $\mathrm{P}$, Bertrand X. Alternatives to carbapenems in ESBL-producing Escherichia coli infections. Med Mal Infect. 2013;43:62-6.

32. Bouxom H, Fournier D, Bouiller K, Hocquet D, Bertrand X. What non-carbapenem antibiotics are active against ESBLproducing Enterobacteriaceae? Int J Antimicrob Agents. 2018;S0924-8579:30091-8.

33. Bassetti M, Peghin M, Pecori D. The management of multidrug-resistant Enterobacteriaceae. Curr Opin Infect Dis. 2016;29:583-94.

34. Bassetti M, Righi E, Carnelutti A, Graziano E, Russo A. Multidrug-resistant Klebsiella pneumoniae: challenges for treatment, prevention and infection control. Expert Rev Anti Infect Ther. 2018;16:749-61.

35. Bassetti M, Vena A, Russo A, Croxatto A, Calandra T, Guery B. Rational approach in the management of $P$. aeruginosa infections. Curr Opin Infect Dis. 2018. https://doi.org/10.1097/qco.00000 00000000505.

36. Samonis G, Maraki S, Karageorgopoulos DE, Vouloumanou EK, Falagas ME. Synergy of fosfomycin with carbapenems, colistin, netilmicin, and tigecycline against multidrug-resistant Klebsiella pneumoniae, Escherichia coli, and Pseudomonas aeruginosa clinical isolates. Eur J Clin Microbiol Infect Dis. 2012;31:695-701.

37. Portier H, Tremeaux JC, Chavanet P, Gouyon JB, Duez JM, Kazmierczak A. Treatment of severe staphylococcal infections with cefotaxime and fosfomycin in combination. J Antimicrob Chemother. 1984;14:277-84.

38. Dinh A, Salomon J, Bru JP, Bernard L. Fosfomycin: efficacy against infections caused by multidrug-resistant bacteria. Scand J Infect Dis. 2012;44:182-9.

39. Pontikis K, Karaiskos I, Bastani S, Dimopoulos G, Kalogirou M, Katsiari M, Oikonomou A, Poulakou G, Roilides E, Giamarellou H. Outcomes of critically ill intensive care unit patients treated with fosfomycin for infections due to pandrug-resistant and extensively drug-resistant carbapenemase-producing Gram-negative bacteria. Int J Antimicrob Agents. 2014;43:52-9.

40. Habib G, Lancellotti P, Antunes MJ, Bongiorni MG, Casalta JP, Del Zotti F, Dulgheru R, El Khoury G, Erba PA, Iung B, Miro JM, Mulder BJ, Plonska-Gosciniak E, Price S, Roos-Hesselink J, Snygg-Martin U, Thuny F, Tornos Mas P, Vilacosta I, Zamorano JL. Task Force per il Trattamento dell'Endocardite Infettiva della Società Europea di Cardiologia (ESC). 2015 ESC Guidelines for the management of infective endocarditis. The Task Force for the Management of Infective Endocarditis of the European Society of Cardiology (ESC)]. G Ital Cardiol (Rome). 2016;17:277-319. 
41. Gennari FJ. Disorders of potassium homeostasis. Hypokalemia and hyperkalemia. Crit Care Clin. 2002;18:273-88.

42. Campion M, Scully G. antibiotic use in the intensive care unit: optimization and de-escalation. J Intensive Care Med. 2018;1:885066618762747.

43. Łój P, Olender A, Ślęzak W, Krzych ŁJ. Pharmacokinetic drug-drug interactions in the intensive care unit-single-centre experience and literature review. Anaesthesiol Intensive Ther. 2017:49:259-67.

44. Finch RG, Eliopoulos GM. Safety and efficacy of glycopeptide antibiotics. J Antimicrob Chemother. 2005;55:ii5-13.

45. Ordooei Javan A, Shokouhi S, Sahraei Z. A review on colistin nephrotoxicity. Eur J Clin Pharmacol. 2015;71:801-10.

\section{Affiliations}

C. Putensen ${ }^{1} \cdot$ B. Ellger ${ }^{2} \cdot$ S. G. Sakka ${ }^{3} \cdot$ A. Weyland ${ }^{4} \cdot$ K. Schmidt $^{5} \cdot$ M. Zoller ${ }^{6} \cdot$ N. Weiler ${ }^{7} \cdot$ D. Kindgen-Milles ${ }^{8}$. U. Jaschinski ${ }^{9} \cdot$ J. Weile ${ }^{10} \cdot$ S. Lindau ${ }^{11} \cdot$ M. Kieninger ${ }^{12} \cdot$ A. Faltlhauser ${ }^{13} \cdot$ N. Jung ${ }^{14} \cdot$ P. Teschendorf ${ }^{15} \cdot$ M. Adamzik ${ }^{16}$.

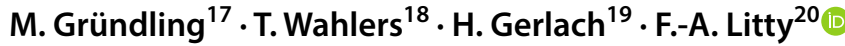

1 Department of Anesthesiology and Surgical Intensive Care Medicine, Medical School of the University of Bonn, Bonn, Germany

2 Department of Anesthesiology, Intensive Care Medicine and Pain Therapy, Klinikum Westfalen GmbH, Dortmund, Germany

3 Department of Anesthesiology and Operative Intensive Care Medicine, Medical Center Cologne-Merheim, University of Witten/Herdecke, Cologne, Germany

4 Department of Anesthesiology/Intensive Care Medicine/Emergency Medicine/Pain Therapy, Klinikum Oldenburg GmbH, Oldenburg, Germany

5 Department of Anesthesiology, Charité University Hospital Berlin, Berlin, Germany

6 Department of Anesthesiology, University Hospital Munich, Munich, Germany

7 Department of Anesthesiology and Intensive Care Medicine, University Medical Center Schleswig- Holstein, Campus Kiel, Kiel, Germany

8 Department of Anesthesiology, University Hospital Düsseldorf, Düsseldorf, Germany

9 Department of Anesthesiology and Surgical Intensive Care Medicine, University Hospital Augsburg, Augsburg, Germany

10 Department of Thorax and Cardiovascular Medicine, Institute of Laboratory and Transfusion Medicine, Heart and Diabetes Center North Rhine-Westphalia, Bad Oeynhausen, Germany
11 Department of Anesthesiology/Intensive Care Medicine/Pain Therapy, University Hospital Frankfurt, Frankfurt, Germany

12 Department of Anesthesiology and Neurosurgical Intensive Care Unit, University Hospital Regensburg, Regensburg, Germany

13 Interdisciplinary Intensive Care Unit, Weiden Hospital, Weiden, Germany

14 Department I of Internal Medicine, University Hospital of Cologne, Cologne, Germany

15 Department of Anesthesiology and Surgical Intensive Care Medicine, Klinikum Osnabrück GmbH, Osnabrück, Germany

16 Department of Anesthesiology/Intensive Care Medicine/Pain Therapy, Knappschaftskrankenhaus Bochum, Bochum, Germany

17 Department of Anesthesiology/Intensive Care Medicine/Emergency Medicine/Pain Therapy, University Hospital Greifswald, Greifswald, Germany

18 Department of Cardiothoracic Surgery, University Hospital of Cologne, Cologne, Germany

19 Department of Anesthesiology and Surgical Intensive Care Medicine, Klinikum Neukölln, Berlin, Germany

20 InfectoPharm Arzneimittel und Consilium GmbH, Heppenheim, Germany 